\title{
Capture of femtosecond plasmon excitation on transient nonequilibrium states of the metal surface
}

\author{
Bo Zhao, ${ }^{1,2}$ Jianjun Yang, ${ }^{1, *}$ Jinluo Cheng, ${ }^{1}$ and Chunlei Guo ${ }^{3}$ \\ ${ }^{1}$ State Key Laboratory of Applied Optics, Changchun Institute of Optics, Fine Mechanics and Physics, \\ Chinese Academy of Sciences, Changchun 130033, China \\ ${ }^{2}$ Department of Electronic Information and Physics, Changzhi University, Changzhi, 046011, China \\ ${ }^{3}$ The Institute of Optics, University of Rochester, Rochester, New York 14627, USA
}

(Received 5 November 2019; revised 7 May 2020; accepted 16 July 2020; published 15 September 2020)

\begin{abstract}
Understanding of laser-material interactions is a scientific evergreen in the fundamental research of physics and optics. We report here that the ultrafast dynamics of the $\mathrm{Cu}(110)$ crystal surface is permanently captured by the formation of subwavelength periodic structures using two collinear femtosecond laser irradiations with different linear polarizations. Surprisingly, such periodic structures are found to have slantwise orientation that is anomalously change as a function of the time delay between two laser beams. In the case of the shorter time delays, the time-dependent slantwise orientations oscillated with terahertz frequency, depending on the pulse width and the intersection angle of two polarization directions, whereas it only presents monotonic change for the larger time delays. Analyses suggests that the former case is attributed to the surface plasmon excitation of the temporally delayed femtosecond laser irradiation on the transient state of the metal surface, which is consequently modulated by some nonthermal effects such as shock wave and bond hardening, while the latter situation is predominated by thermal relaxation of the material lattice. The simulation results agree with the experimental measurements. This investigation not only allows us to sensitively record the transient spatiotemporal evolution on superheated metal surfaces, but also provides insights for the control of material microprocessing.
\end{abstract}

DOI: 10.1103/PhysRevResearch.2.033418

\section{INTRODUCTION}

Understanding the dynamic processes of metal surfaces induced by femtosecond laser irradiation is of importance in both fundamental science and applications, because it is the basis for effective manipulation of material properties at a microscopic level [1,2]. The ultrafast dynamics, such as electron thermalization and the acoustic phonons, generally have been studied using optical transient reflectivity measurements, where the very weak detection is considered from the temporal evolution of the refractive index of materials [3-8]; thus, achieving high signal-to-noise-ratio is often needed with sensitive and sophisticated instruments. In order to improve the detection sensitivity, some pioneering experiments have employed surface plasmons (SPs) as a probe to monitor thermal dynamics in metal films [9-11], in which the timeresolved signals come from the integration on the whole laserexposed surface. However, a comprehensive diagnosis of the ultrafast dynamic processes of the material, such as the spatial change of the refractive index and its physical influence on the subsequent laser actions, is still lacking.

Recently, laser-induced periodic surface structures (LIPSSs) at the subwavelength scale on materials have

\footnotetext{
*jjyang@ ciomp.ac.cn
}

Published by the American Physical Society under the terms of the Creative Commons Attribution 4.0 International license. Further distribution of this work must maintain attribution to the author(s) and the published article's title, journal citation, and DOI. been identified as a universal phenomenon of femtosecond laser-matter interaction especially with the energy fluence near the damage threshold [12-15]. Such technique not only holds the great potential towards nanostructuring of material surfaces, but also demonstrates miraculous effects of the surface refunctionalization [16,17]. Although several mechanisms and pump-probe experiments have been attempted to explain the LIPSSs formation [18-22], the related ultrafast dynamics are not yet clearly depicted. Further deep insights are required for understanding the physical responses of the electrons and lattice that leads to such structures on metal surfaces.

In this paper, we present an approach that utilizes the timedependent orientation change of the subwavelength LIPSSs, to capture the transient nonequilibrium states on copper surface and their physical effects on the surface plamson excitation, under irradiation of two temporally delayed femtosecond laser beams that have collinear propagation but different linear polarizations. With varying time delay of double-laser irradiations, the observed orientation of the LIPSSs tends to change with two different tendencies: terahertz oscillations and monotonic decaying. The discussion and analyses revealed that the former happening only within the time delays less than about $10 \mathrm{ps}$ is physically caused by the noncollinear excitation of surface plasmon on the optically excited metal surface, which suffers the periodic modulations from the round trip of the shock wave within the bond-hardening layer, while the latter occurring at the longer time delays is attributed to the thermal diffusion of the lattice, which makes the transient optical properties gradually decay. The simulation results agree well with the experimental observations. 
(a)

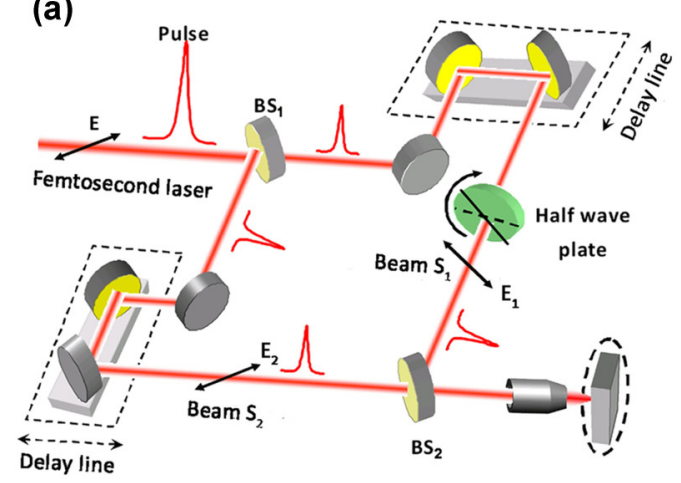

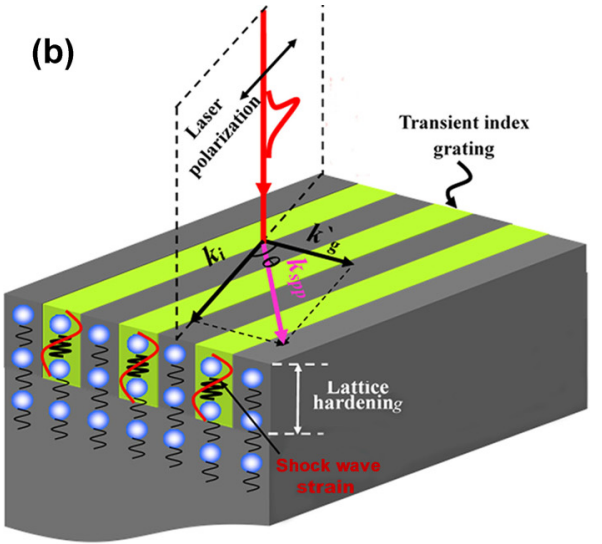

FIG. 1. Schematic diagrams for the observed orientation change of the LIPSSs on copper surface. (a) Experimental setup with two timedelayed collinear femtosecond laser beams linearly polarized in different directions. (b) Sketch of ultrafast dynamic processes occurring on the metal surface upon irradiation of two femtosecond laser beams. The first laser irradiation introduces some transient physical effects, including a transient grating pattern of the optical index (shaded green regions) on the surface and the longitudinal standing shock-wave strain within the bond-hardening layer. This subsequently affects the surface plasmon excitation of the second laser irradiation, thereby changing the spatial orientation of the subwavelength LIPSS (the small springs represent the potential energy force among the metal lattices, which can be increased by the higher electronic temperature).

\section{EXPERIMENTAL METHODS}

The light source in the experiments was a chirped-pulse Ti: sapphire laser amplifier system, which delivered femtosecond laser pulse trains $(1 \mathrm{kHz}, 800 \mathrm{~nm})$ with the horizontal polarization. The laser pulse duration time can be varied from $\tau=50$ fs to 24 ps by adjusting the grating-pair separation in a compressor of the amplifier. As shown in Fig. 1(a), each laser pulse out of the amplifier was divided into two equal-energy beams $\left(S_{1}\right.$ and $\left.S_{2}\right)$ by a splitter $B S_{1}$, and the polarization direction of the laser beam $S_{1}$ was rotated by a half wave plate, leading to an intersection angle of $\theta$ between the two laser polarization directions. After passing through the optical-delay lines, two femtosecond laser beams were adjusted into collinear propagation and focused through a microscope objective lens $(4 \times, \mathrm{NA}=0.1)$ onto the singlecrystal copper (110) surface with a high purity $(>99.99 \%)$ at normal incidence. The surface roughness of the sample was achieved less than $5 \mathrm{~nm}$ through the mechanical and electrochemical polishing treatments. The sample was placed $\sim 300 \mu \mathrm{m}$ before the focus, which allowed for a $1 / e^{2}$ beam spot diameter of approximately $60 \mu \mathrm{m}$ on the target. The experiment was carried out using a line-scribing method (with a scanning speed of $0.1 \mathrm{~mm} / \mathrm{s}$ ) under irradiation of the fixed laser beams, resulting in 600 pulses partially overlapped on one laser spot area. Before and after the experiments, the sample surface was ultrasonically cleaned in acetone solution. The surface morphology of laser-exposed surfaces was examined using a scanning electron microscope (SEM).

\section{EXPERIMENTAL RESULTS}

\section{A. Observation of LIPSSs at zero time-delay irradiation of two lasers}

Figure 2(a) shows the surface morphology of the sample material irradiated by two femtosecond laser beams at zero time delay $(\Delta t=0)$, i.e., the simultaneous illumination of two lasers, where the two laser polarizations are set differently to give an intersection angle of $\theta=45^{\circ}$ (the linear polarization of the laser irradiation $S_{2}$ is always maintained along the horizontal direction), and each laser fluence was sufficiently reduced $\left(F=0.175 \mathrm{~J} / \mathrm{cm}^{2}\right)$ to make no evident damage under its individual irradiation. Clearly, the obtained surface structures exhibit the pattern of one-dimensional subwavelength LIPSSs, associated with a slantwise orientation angle (SOA) of $\alpha \approx 26^{\circ}$ relative to the vertical direction. (Because the single-laser irradiation $S_{2}$ at the higher energy would like to bring the LIPSSs with the vertical orientation, this direction will be considered as a reference for the rest of the paper.) Such structural orientation is totally different from those formed under two individual laser irradiations with the higher energy fluence $\left(F=0.28 \mathrm{~J} / \mathrm{cm}^{2}\right)$, in which each laser irradiation usually interacts with the equilibrium states of the metal surface, resulting in the LIPSS orientation perpendicular to the direction of the laser polarization, as shown in the right panel of Fig. 2(a).The measured SOA approximates one half of the intersection angle $\theta=45^{\circ}$ between two laser polarizations. This result suggests that the LIPSSs formation is based on the physical correlations between two laser-material interaction processes rather than their separated actions. Furthermore, when the intersection angle $\theta$ changes through rotating the polarization direction of the first laser irradiation $S_{1}$, the obtained SOA of the LIPSSs became different; nevertheless, its variation feature follows the dependence of $\alpha \approx \theta / 2$, as shown by the black squares in Fig. 2(b). If the two laser beams have orthogonal polarizations, we can find no LIPSSs formation but some randomly distributed melting nanoparticles on the surface (See Supplemental Material, Fig. S1 [23]). The measured total laser-energy fluencies required for the LIPSSs formation at different intersection angles of $\theta$ are shown by the blue dots in Fig. 2(b), whose agreement with the fitting of $1 / \cos (\theta / 2)^{2}$ (blue dashed curve) indicates the effect of optical interference between the two laser beams. Moreover, we have carried out the experiment to investigate how the 
(a)

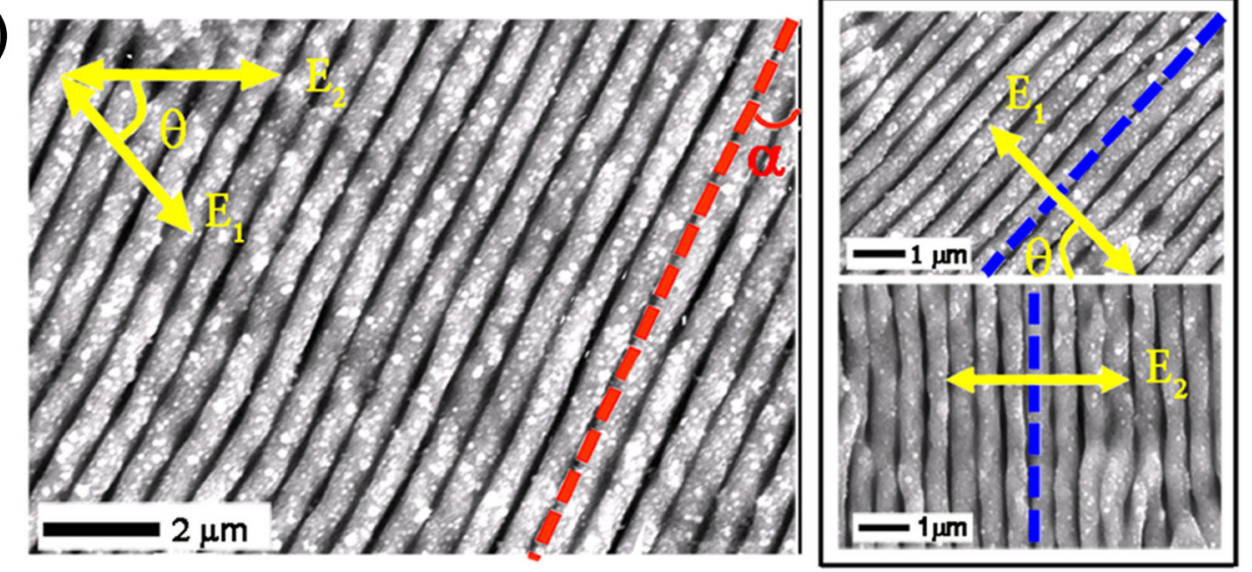

(b)

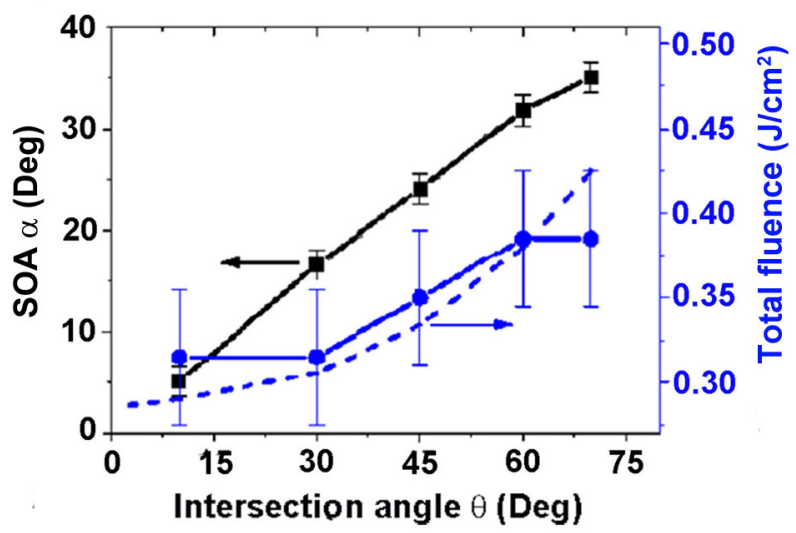

FIG. 2. Formation of the slantwise-oriented LIPSSs by two different linear polarization of femtosecond lasers at zero time-delay irradiation. (a) SEM pictures of the LIPSSs formed on Cu (110) surface by two femtosecond laser irradiations with the intersection angle of $\theta=45^{\circ}$ between their polarization directions (left panel); the results obtained from the two individual laser irradiations with higher energy fluence are also shown (right panel) for comparison, where the yellow double arrows $\left(E_{1}\right.$ and $\left.E_{2}\right)$ denote the electric fields of the laser irradiations; both blue and red dashed lines represent the spatial orientations of the LIPSSs. (b) The measured variation of SOA (black squares) and total energy fluence for the LIPSSs formation (blue circles) as a function of the polarization intersection angle $\theta$, where the blue dashed curve is for the fitting by $1 / \cos (\theta / 2)^{2}$.

LIPSSs change with different intersection angles of $\theta$ when the total fluence was maintained constant. It was found that for the larger $\theta$ values the LIPSSs appearance became faded and even disappeared completely. This is because the intensity of the constructive interference fringes turns to reduce to insufficiently ablate the material surface.

\section{B. Observation of LIPSSs at nonzero time-delay irradiation of two lasers}

In this section we study the dependence of the structural SOA on the time delay of two laser irradiations at a resolution of $200 \mathrm{fs}$, and the results are illustrated in Fig. 3. The variation of SOA of the LIPSSs shows a strong relay on the time delays. For example, at $\Delta t=1.2 \mathrm{ps}$, the measured SOA approximates $\alpha=22^{\circ}$, being $4^{\circ}$ smaller than that of zero time-delay case; at the longer time delay of $\Delta t=2.4 \mathrm{ps}$; however, the measured SOA increases to $\alpha=28.6^{\circ}$ rather than continuing to reduce. In other words, the measured SOA performs an oscillating behavior till the time delay of $\Delta t=12 \mathrm{ps}$, which suggests the complex interplays happening between two laser-material interactions. Nevertheless, for the longer time delays of
$\Delta t>12 \mathrm{ps}$, the measured SOA decreases and it becomes almost zero at $\Delta t>40 \mathrm{ps}$, that is, in this case the LIPSSs are oriented in the vertical direction, indicating the predominant role of the second femtosecond laser irradiation $S_{2}$. Noticeably, when the time delay between two lasers exceeds $\Delta t=$ $80 \mathrm{ps}$, there are no LIPSS formation on the sample surface.

Based on our time-resolved measurements, we can obtain the time-delay dependence of SOA, as depicted in Fig. 4(a). It consists of two features: one is an oscillatory for the short time delays of $\Delta t<12 \mathrm{ps,} \mathrm{which} \mathrm{presents} \mathrm{chirp-frequency}$ oscillations with slow-decaying amplitudes; the other is a monotonic decaying for the longer time delays. Such kind of dependence can be fitted by the following expression:

$$
\begin{aligned}
\alpha= & A \exp \left(-\Delta t / T_{1}\right) \cos [2 \pi f(1-\beta \Delta t) \Delta t+\phi] \\
& +\frac{B}{4\left(\Delta t-t_{c}\right)^{2}+w^{2}}+C,
\end{aligned}
$$

where $A$ and $f$ are the initial values in amplitude and frequency of the oscillations, respectively, $\beta$ for a parameter describing the frequency chirp, $\phi$ for an initial phase; $T_{1}$ for a decay time for the oscillation, $B, t_{c}, w$, and $C$ for the 


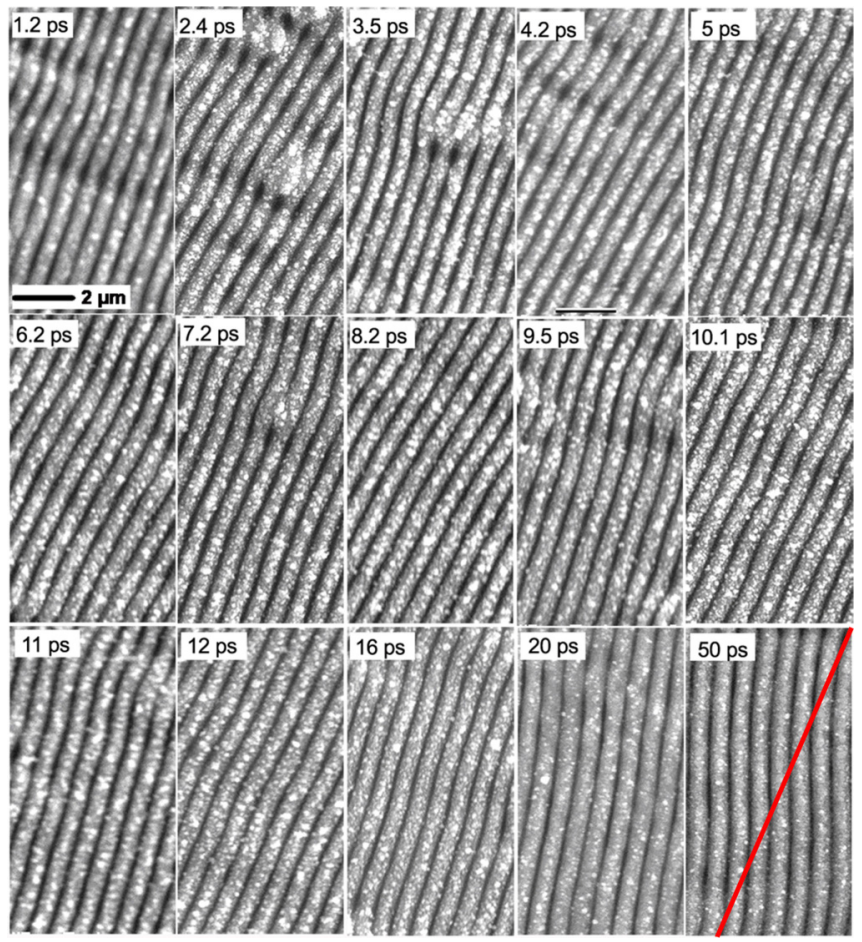

FIG. 3. Observation of the slantwise-oriented LIPSSs on the sample surface for two laser irradiations having an intersection angle of $\theta=45^{\circ}$ between the polarization directions, where the time duration of two lasers is $\tau=50 \mathrm{fs}$ and their total energy fluence is $F=0.35 \mathrm{~J} / \mathrm{cm}^{2}$. The solid red line is for the direction perpendicular to the angular bisector of $\theta$.

parameters describing the slow variation background with a Lorentzian function. As shown in Fig. 4(a), Eq. (1) provides a satisfactory fit (solid red lines) to the experimental data. Figure 4(b) shows the Fourier transform spectra of the measured oscillatory signals in the time domain, and it presents a peak value of approximate $0.53 \mathrm{THz}$. Moreover, it is also seen that the simulation result (solid red curve) can provide a good fitting to the experiment in the frequency domain.

\section{Change of LIPSSs orientation with other laser parameters}

To identify the laser polarization effect on the variation of SOA, we also performed the experiment with other $\theta$ angles, i.e., the polarization direction of the first laser irradiation $S_{1}$ changes while that of the second laser irradiation $S_{2}$ is maintained (see Supplemental Material, Figs. S2-S4 [24]). The measured time-delay responses of the SOA, as shown in Fig. 5(a), also display both the oscillatory and the monotonic decaying components, similar to the case of $\theta=45^{\circ}$. They can also be well fitted using Eq. (1). Here the maximum oscillation magnitude increases from $\Delta \alpha=6^{\circ}$ to $14^{\circ}$ when $\theta$ changes from $10^{\circ}$ to $70^{\circ}$, as illustrated (black squares) in Fig. 5(b). This strongly indicates the physical influence of the ultrafast surface dynamics triggered by the first laser pulse on the final formation of the LIPSSs. In particular, when the two laser polarization directions are set parallel to each other, we can still find the LIPSSs but with the constant SOA regardless of the time delays, which is equivalent to a zero in the oscillation magnitude. Moreover, the initial value of the oscillation frequency $f$ is also found to have an increasing tendency with the increase of $\theta$, as shown (blue dots) in Fig. 5(b).

Furthermore, the time-delay dependence of the SOA oscillation is also strongly affected by the incident laser pulse width (see Supplemental Material, Figs. S5 and S6 [25]). For example, for the case of $\theta=45^{\circ}$ when the laser pulse width is stretched to $\tau=1 \mathrm{ps}$, the measurement of SOA presents the oscillatory behavior for the time delays less than $\Delta t=11 \mathrm{ps}$ and then it is followed by the monotonic decaying process, which is similar to the observations made by the 50-fs laser pulses. Under such circumstance, both the maximum magnitude and the frequency of the SOA oscillation are decreased to $\Delta \alpha=5^{\circ}$ and $f=0.3 \mathrm{THz}$, respectively, as shown in Fig. 5(c). In other words, increasing the laser pulse width results in a redshifted oscillation frequency for the SOA change. When the laser pulse width was further stretched to $\tau=10 \mathrm{ps}$, the measured oscillating behaviors in the SOA change become rather indistinct within the shorter time-delay range, whereas the monotonic decaying change of the SOA still remains for the larger time delays.
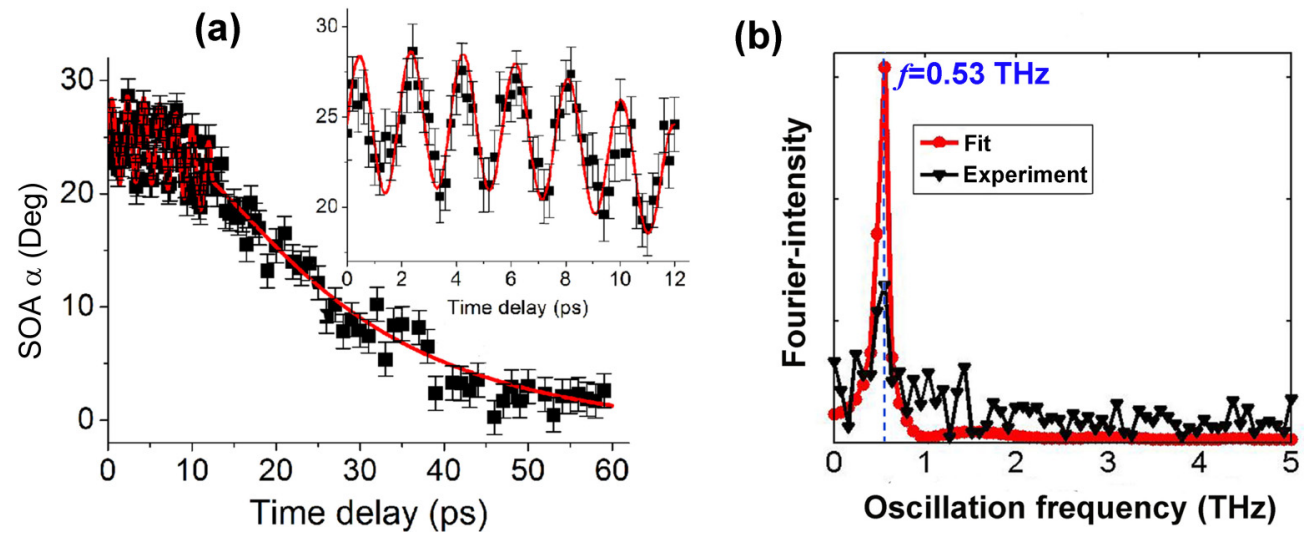

FIG. 4. (a) Evolution of the SOA of the LIPSSs with the time delay between two femtosecond laser irradiations $\left(\tau=50 \mathrm{fs}, \theta=45^{\circ}\right)$, where the solid black squares represent the experimental data, and the solid red line is for the fitting curve. The inset picture shows the details of the oscillatory component within the time delays less than $\Delta t=12 \mathrm{ps}$. (b) The obtained Fourier transform spectra for the measured oscillations in time domain, where the red line represents a numerical fitting based on Eq. (1). 


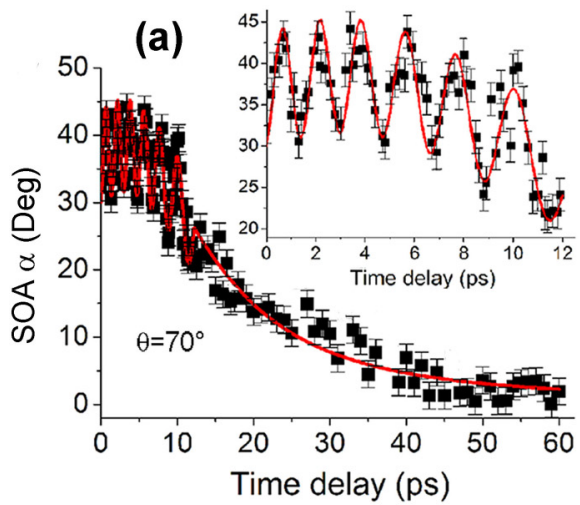

(b)

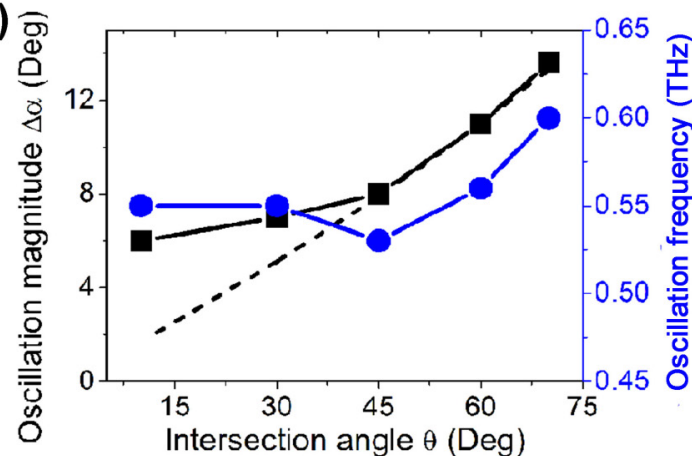

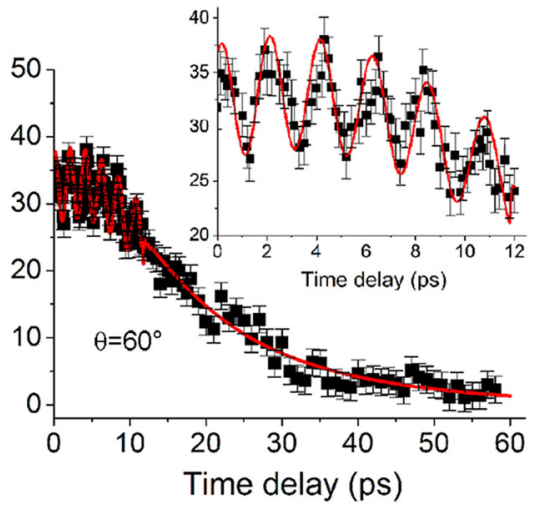

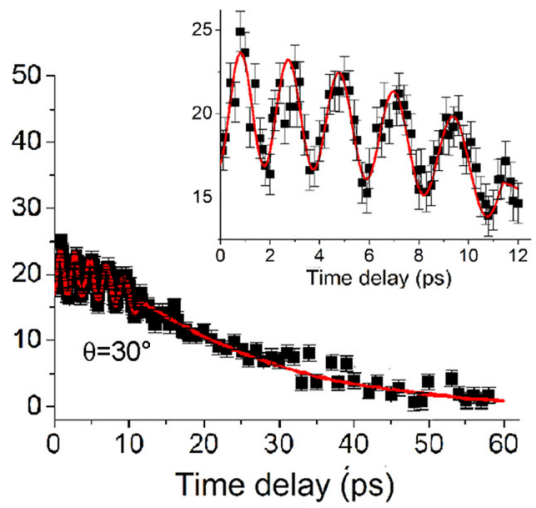

(c)

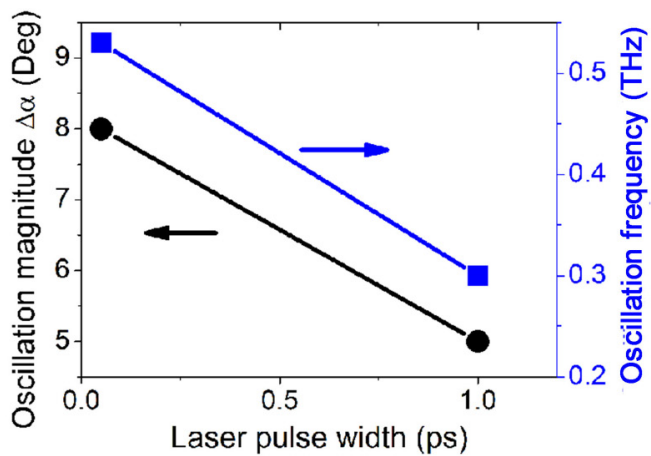

FIG. 5. Measured influence of the other laser parameters on the oscillation change of the SOA. (a) Time-delay-dependent oscillations of the SOA induced by different polarization intersection angles of $\theta=70^{\circ}, 60^{\circ}$, and $30^{\circ}$, where the total laser energy fluences are $F=0.385 \mathrm{~J} / \mathrm{cm}^{2}$, $0.385 \mathrm{~J} / \mathrm{cm}^{2}$, and $0.315 \mathrm{~J} / \mathrm{cm}^{2}$, respectively. The solid black squares represent the experimental data, and the solid red lines are for the fitting curves. (b) Dependence of the maximum magnitude (black squares) and the frequency (blue circles) for the SOA oscillation on the $\theta$ angle. The dashed line represents the simulation result of Eq. (3). (c) Dependence of the maximum magnitude (black circles) and the frequency (blue squares) for the SOA oscillation on the laser pulse width.

\section{DISCUSSION AND ANALYSES}

For the case of zero time-delay irradiation, the simultaneous irradiation of two lasers provides conditions for the superposition of their electric fields, leading to a synthesized vector along the angular bisector direction of $\theta$, which is equivalent to a new single-laser irradiation. According to the previous studies [13-15,26], the corresponding surface plasmon excitation (with a wave vector $\overrightarrow{k_{s p}}$ ) and its subsequent interference with the scattering light on the surface (with a wave vector $\overrightarrow{k_{i}}$ ) results in the distribution of the intensity fringes (with a periodicity $\Lambda$ ), whose ablation of the material surface brings forth the periodic grooves (with a grating vector $\left|\vec{k}_{g}\right|=2 \pi / \Lambda$ parallel to the electric field direction). The physical correlation among the aforementioned processes can be described by $\overrightarrow{k_{g}}=\overrightarrow{k_{s p}}-\overrightarrow{k_{i}}$. Usually, the directions of such three vectors are parallel to each other, so that the LIPSSs orientation is perpendicular to the laser polarization. In an alternative understanding, the orientation of the LIPSSs is actually determined by the direction of the excited SP vector. Therefore, the structure orientation induced by two zero timedelay laser irradiations has a slantwise degree of $\alpha=\theta / 2$ with respect to the vertical direction, which is consistent with the experimental observations.

For the case of nonzero time-delay irradiations, the optical interference between two laser irradiations becomes substantially impossible, thus the underlying mechanisms for the structure formation are different from those of zero timedelay situations. Considering the time-independent parameters of two laser irradiations, the orientation change of LIPSSs can be reasonably attributed to the interplay of the second laser irradiation $S_{2}$ with the nonequilibrium properties of the metal surface triggered by the first laser irradiation $S_{1}$. The relevant physical processes can be depicted as follows: Being similar to the single-beam laser irradiation, the first laser irradiation $S_{1}$ generates the intensity fringes due to its $\mathrm{SP}$ excitation, the subsequent absorption of which modifies the optical index of the metal surface into the periodic gratinglike patterns (with a grating vector $\overrightarrow{k_{g}^{\prime}}$ parallel to the polarization direction of the laser irradiation $S_{1}$ ) [19,20]. In physics, the translational symmetry of the metal surface is thus destroyed. Because of the short-life existence, such obtained periodic patterns of the optical index earn the appellation "transient grating of the refractive index." By standing at the point of the time-delayed laser irradiation $S_{2}$, we can consider that there exists an azimuth angle of $\theta$ between its polarization direction and the transient grating vector $\overrightarrow{k_{g}^{\prime}}$. According to Ref. [27], the noncollinear directions of $\overrightarrow{k_{i}}$ and $\overrightarrow{k_{g}^{\prime}}$ vectors can modulate the vectorial direction of SP excitation, which consequently determines the spatial orientation of the LIPSSs. The coupling condition is described using a simple trigonometry, i.e., 


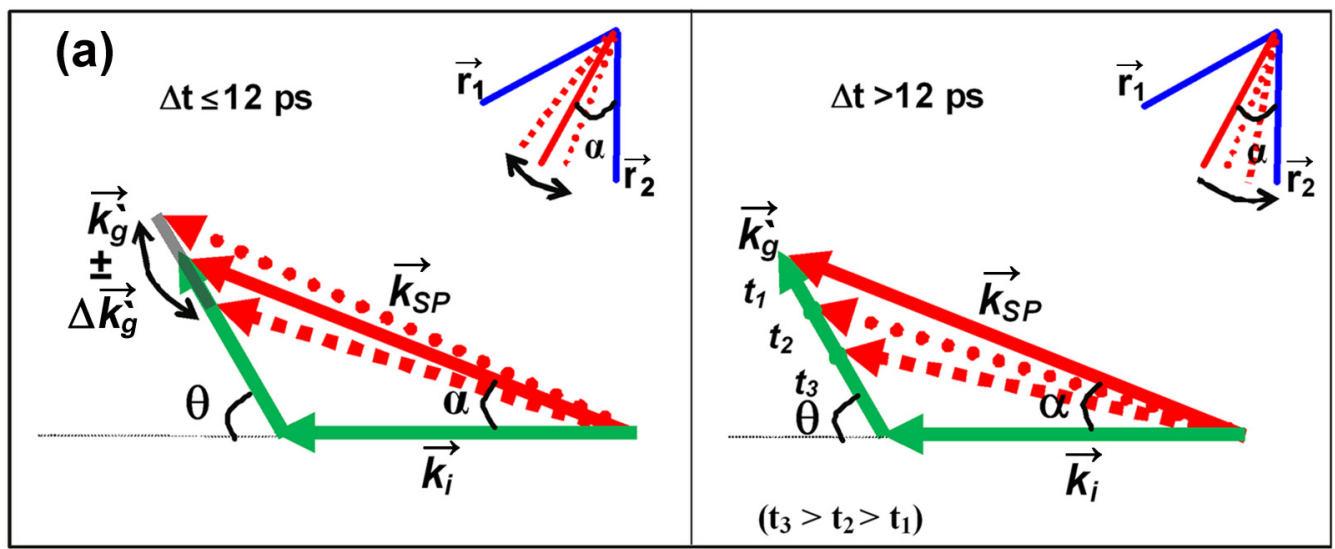

(b)

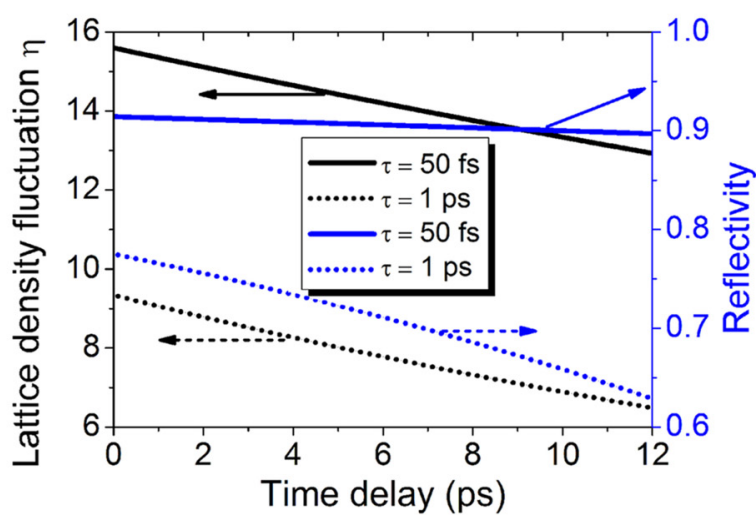

FIG. 6. Theoretical analyses of the temporal evolution of the LIPSSs orientation on copper surfaces. (a) Proposed physical model for the observed SOA change by two femtosecond laser irradiations with the linear polarization in different directions (left and right panels represent different time-delay regimes), where $\Delta \overrightarrow{k_{g}^{\prime}}$ represents the modulation of the transient grating vector based on the shock-wave effect, and $\overrightarrow{k_{s p}}$ (red lines) for the excited SP vector of the second laser irradiation. Inset pictures located at the upper-right corner of each panel map the physical process of the SOA oscillation, with $\overrightarrow{r_{1}}$ and $\overrightarrow{r_{2}}$ being the structure orientations formed by two individual high energy fluence of laser irradiations $S_{1}$ and $S_{2}$, respectively. (b) Simulation results of the lattice density fluctuation (black lines) and the shock-wave reflectivity (blue lines) at the boundaries of bond hardening for two different laser pulse widths.

$\overrightarrow{k_{s p}}=\overrightarrow{k_{i}}+\overrightarrow{k_{g}^{\prime}}$, as shown in Fig. 6(a). Based on the geometrical relation, the slantwise degree of $\overrightarrow{k_{s p}}$ excited from the delayed laser irradiation $S_{2}$ is given by

$$
\tan \alpha=\frac{\left|\overrightarrow{k_{g}^{\prime}}\right| \sin \theta}{\left|\overrightarrow{k_{i}}\right|+\left|\overrightarrow{k_{g}^{\prime}}\right| \cos \theta} .
$$

Accordingly, the observed time-dependent change of the SOA can be attributed to the modulation on the transient grating vector to bring about $\Delta \overrightarrow{k_{g}^{\prime}}(\Delta t)$. Now we can rewrite Eq. (2) into a new expression:

$$
\tan (\alpha+\Delta \alpha)=\frac{\left|\overrightarrow{k_{g}^{\prime}} \pm \Delta \overrightarrow{k_{g}^{\prime}}\right| \sin \theta}{\left|\overrightarrow{k_{i}}\right|+\left|\overrightarrow{k_{g}^{\prime}} \pm \Delta \overrightarrow{k_{g}^{\prime}}\right| \cos \theta}
$$

Clearly, $\Delta \alpha$ tends to increase with increasing $\theta$ at the given $\Delta \overrightarrow{k_{g}^{\prime}}$, and from its periodic change with the time delay one can deduce a time-delay-dependent periodic change of $\Delta \overrightarrow{k_{g}^{\prime}}(\Delta t)$ which is easier to reveal the physical essence of the SOA oscillations. The calculated oscillation magnitude of the SOA versus different $\theta$ angles is shown in Fig. 5(b), which matches the experimental data.

Physically, the periodic time-delay variation of $\Delta \overrightarrow{k_{g}^{\prime}}(\Delta t)$ can be understood from the time-delay change in the dielectric constant $\varepsilon_{m}(\Delta t)$ of the transient index grating, which is described as follows: When the first laser irradiation $S_{1}$ hits the target to result in the SP excitation and the transient index grating, the electrons in the local periodic energy deposition areas are overheated and consequently produce a pressure or stress through potential deformation [28]. As a result, the equilibrium position of the lattice is altered, and it subsequently affects the optical index of the transient grating vector. This ultrafast laser-induced stress is proportional to the electronic temperature change of $\delta T_{e}(z, t)$, i.e., $\sigma_{D P}=$ $-\gamma_{e} C_{e} \delta T_{e}(z, t)\left(\gamma_{e}\right.$ and $C_{e}$ being the Grüneisen coefficient and heat capacity of electrons, respectively [28]), and can generate shock wave propagating along the depth direction [29-31] (see Supplemental Material, Fig. S7 for the temperature evolution of both the electrons and the lattice, where the electron temperature is heated up to several tens of thousands of kelvin within the relaxation time of $\Delta t=12 \mathrm{ps}$ while the 
lattice temperature is kept virtually unperturbed [32]). On the other hand, the previous studies proved that such transient extreme conditions can also cause a bond-hardening layer on the metal surface through reducing the screening of the attractive interatomic potential by delocalization of highly excited free electrons [33-36], thus the metal properties are inevitably modulated by the increase of the lattice stability, e.g., producing a higher melting temperature and a larger elastic stiffness, and making the shock-wave velocity $(v)$ differ from that of the unaffected regions. Eventually, the mismatch of the shock-wave impedance can be achieved at the boundaries of bond-hardening layer (a thickness $L$ ) to cause the reflection of shock wave, which is very similar to the observations of the periodic acoustic signals in ultrafast heating of metal films deposited on the substrate material [12,37]. In other words, it is the shock-wave generation and bond hardening induced by the first laser irradiation that result in the round trips of the longitudinal shock wave with the frequency of $v / 2 L$, leading to the modulation of the transient optical properties of the material surface at the same frequency. This kind of shock wave happening within the time range of the electron-lattice coupling belongs to the nonthermal behavior.

Because the formation of standing shock wave within the bond-hardening layer creates periodic fluctuations in the lattice density, i.e., $\eta\left(t-\frac{2 L}{v}\right)=\left[n_{l}\left(t-\frac{2 L}{v}\right)-n_{l}^{0}\right] / n_{l}^{0}$, where $n_{l}\left(t-\frac{2 L}{v}\right)$ and $n_{l}^{0}$ are the lattice density in the nonequilibrium and the equilibrium states, respectively [38], the timedependent dielectric function on the metal surface can be written as $\varepsilon_{m}\left(t-\frac{2 L}{v}\right)=\varepsilon_{m}^{0}\left[1+n_{l}\left(t-\frac{2 L}{v}\right)\right]$ with $\varepsilon_{m}^{0}$ being the equilibrium-state dielectric function, and consequently modulates the transient grating vector $\left(\overrightarrow{k_{g}^{\prime}}\right)$ by $\Delta \overrightarrow{k_{g}^{\prime}}\left(t-\frac{2 L}{v}\right)=$ $\frac{\vec{k}_{i}}{2 \mid \varepsilon_{m}^{0}} \eta\left(t-\frac{2 L}{v}\right)$. Therefore, the vectorial direction of the excited $\mathrm{SP}$ for the second laser irradiation $S_{2}$ is periodically altered as a function of the time delay, as shown in Fig. 6(a) (left panel), and leads to the oscillation change of the structure orientation.

Now we give some estimation based on the mechanism discussed above. From the linear absorption data of copper in Ref. [39], a penetration depth approximates as $12 \mathrm{~nm}$ at the wavelength of $800 \mathrm{~nm}$, which suggests that the thickness of bond-hardening layer is roughly limited within such optically excited region. Therefore, with the measured average oscillation frequency of $0.53 \mathrm{THz}$ for the SOA of the structures at $\theta=45^{\circ}$, we obtain a velocity of $v=12.7 \mathrm{~km} / \mathrm{s}$ for the shock wave propagating inside the transient bond-hardening layer, about 2.7 times higher than the sound velocity of $4.7 \mathrm{~km} / \mathrm{s}$ in copper material. This calculation result seems to be reasonable compared with the report in Ref [29], where the shock-wave velocity in crystalline iron material excited by femtosecond laser is 4 times higher than the tabulated value at room temperature. Accordingly, for the higher electron temperature, larger shock-wave velocity is expected, which corresponds to a larger oscillation frequency of SOA. This prediction can be confirmed by the measured data (blue squares) in Fig. 5(b), where higher incident laser fluences are required for larger $\theta$. Moreover, with increasing time delays, the laser-induced bond-hardening effect on the metal surface is gradually weakened via the electron-lattice coupling that dissipates the electron energy within a few tens of picoseconds, which makes the shock-wave velocity slower; simultaneously, the electron diffusion process that carries the energy farther into the depth can extend the thickness of the bond-hardening layer. Both increase the round-trip time of shock-wave propagation, being consistent with the observed decreasing frequency of the SOA oscillation. With the electron temperature reduced and the lattice temperature increased, their thermal equilibrium is reached at approximately $12 \mathrm{ps}$, at which the lattice is softened to make the boundaries of bond hardening become indistinct [31]; thus, the round trip of shock wave cannot be supported anymore. Correspondingly, there is no occurring of the periodic disturbance on the lattice density. This can explain the disappearing SOA oscillation at the time delay of 12 ps in Fig. 4(a).

When the time delay is longer than the electron-lattice relaxation, the temporal evolution of the transient grating is dominated by thermal diffusion, which makes the transient grating vector $\left(\overrightarrow{k_{g}^{\prime}}\right)$ gradually reduce. Consequently, the vectorial direction of the excited SP of the second laser irradiation $S_{2}$ monotonically changes toward the laser wave vector $\overrightarrow{k_{i}}$, as shown in Fig. 6(a) (right panel). This is why the decaying variation of the SOA occurs during the time interval of 12 $40 \mathrm{ps}$. For time delay longer than $40 \mathrm{ps}$, the transient grating dissipates to disappear completely, so that the SOA no longer changes.

When the pulse width of two laser irradiations was stretched into picoseconds with the reduced peak intensities, the level of electron temperature improvement would likely be decreased, which of course weakens the bond-hardening strength in the metal surface. The longer the pulse width, the lower the electron temperature and the weaker the bond hardening becomes. As a result, the shock-wave velocity within the bond-hardening layer turns out to be smaller. The simulations of the pulse-width-dependent physical effects are identified in Fig. 6(b) (see details in Supplemental Material [40]), wherein the stronger deformation potential induced by the femtosecond laser causes both the larger fluctuations in the lattice density and the greater mismatch in the shock-wave velocity; thus, the higher reflection of approximately $90 \%$ for the shock wave arriving at the bond-hardening boundaries is obtained and it can remain during the electron-lattice relaxation process. We can also see from Fig. 6(b) that for the incident laser pulse width of $1 \mathrm{ps}$ the available shock-wave reflection at the bond-hardening boundaries decreases to approximately $70 \%$ due to the smaller fluctuation in the lattice density, leading to smaller variations of $\Delta \overrightarrow{k_{g}^{\prime}}$, which finally presents the weaker change in the SOA oscillation. In other words, when the laser intensity reduces at the longer pulse width, the level of bond hardening is weakened and the round-trip time of the shock-wave propagation is apt to increase, resulting in the lower frequency $(0.3 \mathrm{THz})$ of the SOA oscillation, as shown in Fig. 5(c). Correspondingly, the calculated shockwave velocity in the bond-hardening condition approximates $v=6 \mathrm{~km} / \mathrm{s}$, slightly larger than the sound velocity. If the pulse widths of the laser irradiations are stretched more than $10 \mathrm{ps}$, a small increase of the electron temperature makes the bond hardening negligible and thus the round trip of 
the shock wave rarely occurs, leading to no change of SOA oscillation.

\section{CONCLUSIONS}

In summary, we presented observation of the time-resolved modulations on the subwavelength LIPSS formation on copper surface using two different linearly polarized femtosecond laser irradiations. The experimental results discovered two distinct variation tendencies for the evolution of the structure orientation with the time delay between two lasers: the terahertz-frequency oscillations for the time delays less than 12 ps and the monotonic decaying at longer time delays. Moreover, it was also found that the oscillation behaviors are more likely affected by both the laser pulse width and the polarization direction. The involvement of physics has been revealed as follows: During the oscillation regime, the incident first laser irradiation firstly produces the transient gratinglike distribution of the refractive index on the metal surface, along with other nonthermal effects such as shock wave and bond hardening. Because of their periodic physical disturbances and modulations, the noncollinear excitation of surface plasmon for the time-delayed second laser irradiating on the metal surface occurs, which consequently changes the LIPSS orientation in a periodic way. For the case of monotonic decaying observation, the time-dependent evolution of the transient grating was dominated by thermal diffusion of the material, which makes the transient grating effect gradually vanish. Our physical analyses presented satisfactory explanations to the experimental phenomena, with good consistency between the simulation and the measurement results. We believe that the further investigation into this line of research will not only give deeper insights into the ultrafast dynamic processes of femtosecond laser-metal interactions but also benefit metal processing and manufacture.

\section{ACKNOWLEDGMENTS}

We thank Mr. Fang Xie for the preparatory experimental work. We acknowledge financial support from National Natural Science Foundation of China (Grants No. 91750205 and No. 11674178), Jilin Provincial Science \& Technology Development Project (Grant No. 2018414019GH), Scientific and Technological Innovation Programs of Higher Education Institutions in Shanxi (Grant No. 2019L0902), and K. C. Wong Education Foundation (Grant No. GJTD-2018-08).
[1] E. D. Potter, J. L. Herek, S. Pedersen, Q. Liu, and A. H. Zewail, Nature (London) 355, 66 (1992).

[2] M. Wolf, Surf. Sci. 343, 377 (1997).

[3] C. K. Sun, F. Vallée, L. Acioli, E. P. Ippen, and J. G. Fujimoto, Phys. Rev. B 48, 12365 (1993).

[4] N. Del Fatti, R. Bouffanais, F. Vallée, and C. Flytzanis, Phys. Rev. Lett. 81, 922 (1998).

[5] M. Hase, K. Ishioka, J. Demsar, K. Ushida, and M. Kitajima, Phys. Rev. B 71, 184301 (2005).

[6] O. V. Misochko, M. Hase, K. Ishioka, and M. Kitajima, Phys. Rev. Lett. 92, 197401 (2004).

[7] O. B. Wright and K. Kawashima, Phys. Rev. Lett. 69, 1668 (1992).

[8] A. Stolow, A. E. Bragg, and D. M. Neumark, Chem. Rev. 104, 1719 (2004).

[9] M. van Exter and A. Lagendijk, Phys. Rev. Lett. 60, 49 (1988).

[10] J. Wang and C. Guo, Phys. Rev. B 75, 184304 (2007).

[11] V. Temnov, K. Nelson, G. Armelles, A. Cebollada, T. Thomay, A. Leitenstorfer, and R. Bratschitsch, Opt. Express 17, 8423 (2009).

[12] J. Reif, O. Varlamova, and F. Costache, Appl. Phys. A 92, 1019 (2008).

[13] A. Borowiec and H. K. Haugen, Appl. Phys. Lett. 82, 4462 (2003).

[14] L. Xue, J. Yang, Y. Yang, Y. Wang, and X. Zhu, Appl. Phys. A 109, 357 (2012).

[15] A. Vorobyev, V. Makin, and C. Guo, J. Appl. Phys. 101, 034903 (2007).

[16] A. Y. Vorobyev and C. Guo, Laser Photon. Rev. 7, 385 (2013).

[17] H. Qiao, J. Yang, F. Wang, Y. Yang, and J. Sun, Opt. Express 23, 26617 (2015).

[18] G. Miyaji and K. Miyazaki, Opt. Express 16, 16265 (2008).

[19] J. Bonse, A. Rosenfeld, and J. Krüger, J. Appl. Phys. 106, 104910 (2009).
[20] S. Hohm, A. Rosenfeld, J. Kruger, and J. Bonse, Appl. Phys. Lett. 102, 054102 (2013).

[21] X. Zhou, X. Jia, T. Jia, K. Cheng, K. Cao, S. Zhang, D. Feng, and Z. Sun, J. Appl. Phys. 121, 104301 (2017).

[22] R. Fang, A. Vorobyev, and C. Guo, Light Sci. Appl. 6, e16256 (2017).

[23] See Supplemental Material at http://link.aps.org/supplemental/ 10.1103/PhysRevResearch.2.033418 for details on SEM image of surface structures induced by two orthogonally polarized 50fs laser beams at zero time delay in Fig. S1.

[24] See Supplemental Material at http://link.aps.org/supplemental/ 10.1103/PhysRevResearch.2.033418 for details on SEM images of periodic structures induced by two time-delayed 50fs laser beams at variable intersecting polarization angles in Figs. S2-S4.

[25] See Supplemental Material at http://link.aps.org/supplemental/ 10.1103/PhysRevResearch.2.033418 for details on the temporal evolution of the structural SOA induced by two laser beams with broader pulse widths in Figs. S5 and S6.

[26] K. Okamuro, M. Hashida, Y. Miyasaka, Y. Ikuta, S. Tokita, and S. Sakabe, Phys. Rev. B 82, 165417 (2010).

[27] S. J. Elston, G. P. Bryan-Brown, and J. R. Sambles, Phys. Rev. B 44, 6393 (1991).

[28] P. Ruello and V. E. Gusev, Ultrasonic 56, 21 (2015).

[29] Y. Hirayama, P. A. Atanasov, M. Obara, N. N. Nedialkov, and S. E. Imamova, Jpn. J Appl. Phys. 45, 792 (2006).

[30] M. E. Povarnitsyn, T. E. Itina, M. Sentis, K. V. Khishchenko, and P. R. Levashov, Phys. Rev. B 75, 235414 (2007).

[31] D. S. Ivanov and L. V. Zhigilei, Phys. Rev. Lett. 91, 105701 (2003).

[32] See Supplemental Material at http://link.aps.org/supplemental/ 10.1103/PhysRevResearch.2.033418 for details on simulated temporal evolution of the electron and lattice temperatures for the copper surface irradiated by the first incident 
laser beam $S_{1}$ with the energy fluence of $F=0.175 \mathrm{~J} / \mathrm{cm}^{2}$ (Fig. S7).

[33] F. Cheenicode Kabeer, E. S. Zijlstra, and M. E. Garcia, Phys. Rev. B 89, 100301(R) (2014).

[34] R. Ernstorfer, M. Harb, C. T. Hebeisen, G. Sciaini, T. Dartigalongue, and R. J. D. Miller, Science 323, 1033 (2009).

[35] V. Recoules, J. Clerouin, G. Zerah, P. M. Anglade, and S. Mazevet, Phys. Rev. Lett. 96055503 (2006).

[36] B. I. Cho, K. Engelhorn, A. A. Correa, T. Ogitsu, C. P. Weber, H. J. Lee, J. Feng, P. A. Ni, Y. Ping, A. J. Nelson et al., Phys. Rev. Lett. 106, 167601 (2011).
[37] N. Nakamura, H. Ogi, and M. Hirao, Phys. Rev. B 77, 245416 (2008).

[38] V. Temnov, C. Klieber, K. Nelson, T. Thomay, V. Knittel, A. Leitenstorfer, D. Makarov, M. Albrecht, and R. Bratschitsch, Nat. Commun. 4, 1468 (2013).

[39] M. Lejman, V. Shalagatskyi, O. Kovalenko, T. Pezeril, V. V. Temnov, and P. Ruello, J. Opt. Soc. Am. B 31, 282 (2014).

[40] See Supplemental Material at http://link.aps.org/supplemental/ 10.1103/PhysRevResearch.2.033418 for details on calculation of the lattice density fluctuation and the acoustic reflectivity at the boundaries of the lattice-hardening layer. 